\title{
Dynamic Virtual Environments Using Really Simple Syndication
}

\author{
Andrew Dunk, Ronan Jamieson, and Vassil Alexandrov \\ Centre for Advanced Computing and Emerging Technologies \\ The University of Reading \\ a.dunk@reading.ac.uk \\ http://www.acet.reading.ac.uk
}

\begin{abstract}
This paper investigates the use of really simple syndication (RSS) to dynamically change virtual environments. The case study presented here uses meteorological data downloaded from the Internet in the form of an RSS feed, this data is used to simulate current weather patterns in a virtual environment. The downloaded data is aggregated and interpreted in conjunction with a configuration file, used to associate relevant weather information to the rendering engine. The engine is able to animate a wide range of basic weather patterns. Virtual reality is a way of immersing a user into a different environment, the amount of immersion the user experiences is important. Collaborative virtual reality will benefit from this work by gaining a simple way to incorporate up-todate RSS feed data into any environment scenario. Instead of simulating weather conditions in training scenarios, actual weather conditions can be incorporated, improving the scenario and immersion.
\end{abstract}

Keywords: Virtual Reality, Weather, RSS, XML, OpenSG.

\section{Introduction}

Virtual Reality is a way of showing information to users in such a way that it can be interacted with, as you would expect to interact with the real world. However it is not limited to this, it also allows users to view fictional items or objects that would not be possible to visualise easily in the real world. The most popular method for achieving a virtual reality is to use three dimensional visualisations for the users to view. The senses of touch (haptics), sound, smell and even taste have been experimented with as well.

A CAVE [4 is a fully immersive 3D environment able to display stereo imagery that can span 4 walls, the floor and ceiling, giving the user the impression that they are physically in the virtual environment being displayed. Presence is a measure of how accepting a user is to a virtual environment, or how detached they are from the real world. By bringing real world information into the virtual one like the current weather, or information that users are accustomed to, like a mobile phone telephone book, helps users to accept the virtual environment, increasing their presence. 
This work is a continuation of the Virtual Weather [5] project, which aimed to create a virtual reality environment to simulate some basic weather types over a loaded model. This work will concentrate and generalise the area of downloading and parsing data from the Internet, by implementing a suitable Extensible Markup Language (XML) parser to be used for both configuring the application and parsing any RSS data downloaded from the Internet. This generalisation enables relationships of any RSS data to variables of a rendering engine. Including but not limiting the application to downloading different weather sources and rendering them into a virtual environment. Fig 1 shows the result of an RSS weather feed downloaded from the Internet and its representation rendered in a CAVE.

Collaborative virtual environments (CVE), scenario training, product searching, and other real world data related applications can benefit from this work by gaining a simple way to incorporate up-to-date RSS feed data into their systems. The ability to dynamically change environment variables from RSS feeds enables a vast amount of information to be incorporated into a CVE, increasing immersion, productivity, and quality of the system.

Another goal for the project is platform independance, to allow the application to run on multiple architectures.

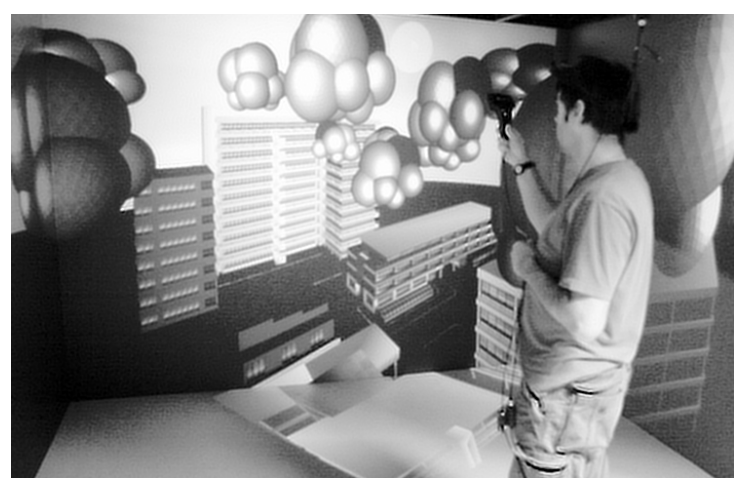

Fig. 1. Downloaded RSS weather data representation rendered in the CAVE

\section{Related Work and Background}

There is a large amount of work in rendering weather and animating weather conditions, including photo-realistic weather animations in real time. Modern day computer games are becoming involved in using more realistic weather simulations, quite recently Second Life [7] has just started improving its environment by integrating a new photo realistic weather rendering system [6] from Windward Mark Interactive [1] called Nimble ${ }^{\mathrm{TM}}$ and WindLight ${ }^{\mathrm{TM}}$ which use physics based algorithms for rendering volumetric clouds and calculating the effects the atmosphere has on a lighting model. The Second Life game environment has 
simulated weather patterns that change during play but the weather information is generated for the game environment and does not relate to the real world weather. This must not be confused with the 3D Weather Data Visualization in Second Life [1] which is an in game 3D map of the United States of America with up-to-date weather data of America.

Architects use similar technologies to view their designs in the real world, enabling them to see casting shadows and other aesthetics that may be difficult to visualise normally. These systems can simulate the effects of the sun during any time of the day, but do not gather weather information from the Internet.

There are a few games that support real world weather conditions, these include some of the more recent flight simulators like the Microsoft Flight Simulator [9], and Black \& White [10] a real time strategy game. Both these games use dedicated weather information that is specifically formed to work with the application and is downloaded from a dedicated server. In Black \& White you are able to simulate a weather location by typing in your zip or postal code into the game, and Microsoft Flight Simulator simulates up-to-date weather of the location you are flying in.

A mobile phone application named Mobile Weather [3] uses Yahoo Weather 12 RSS feeds to display location based weather information to the user on their mobile phone but does not render the data in any way, it displays the data in an easy to read format.

\section{Overview of Approach}

This work differs from other work by having the ability to use any RSS data source, linking select information with a rendering engine. This section describes the integral sections of the work and how they combine together with a rendering engine. The use case describes this projects use in rendering a weather simulation. Fig 2 shows a basic representation of the systems structure.

\subsection{RSS Downloader}

All underlying networking used to connect to the Internet and download RSS feeds is achieved through the use of sockets and a very basic set of HTTP 1.1 protocol [8] commands. Connections to a web server can also be made via a proxy with or without basic authentication, the proxy server settings are set in the configuration file for the application. Sensitive data such as proxy authentication can be left blank in the configuration file, the application will ask for these details at runtime.

When downloading an RSS feed, first a connection request is made to the specified server, on success a page request of a known RSS feed is sent and the response is stored into memory, the RSS data is retrieved from this response and passed to the XML parser. 


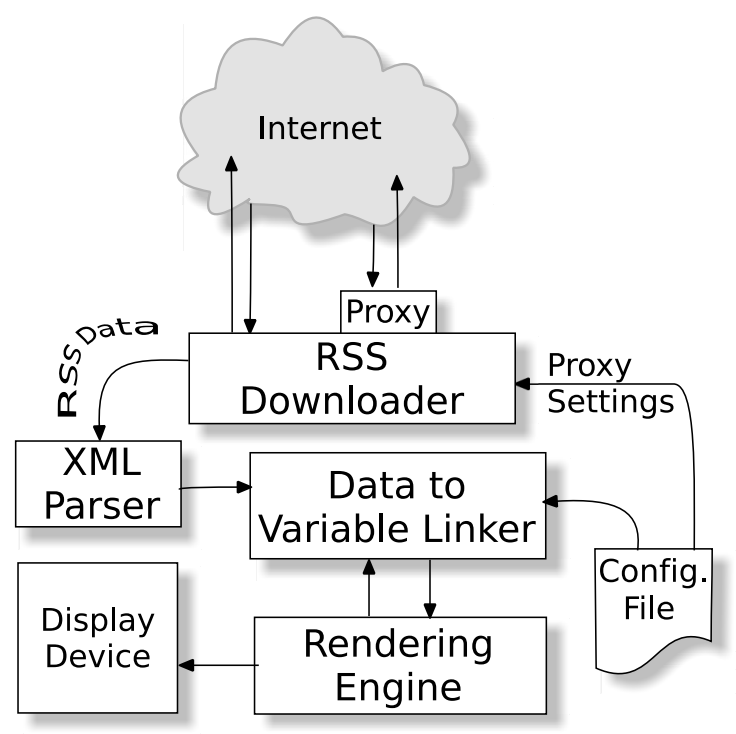

Fig. 2. A System Overview

\subsection{XML Parser}

RSS feeds are written using XML, therefore a very small, fast and effective, open-source, cross platform $\mathrm{C}++\mathrm{XML}$ parser $[2$ is used within the application to both parse the downloaded RSS feeds, and the configuration files that help link the RSS feed data to relevant variables in the rendering engine as well as general configuration options for the application itself.

The XML parser loads a full XML file into memory, this can either be from physical file or memory location, it parses the data and generates a tree structure representing the XML file. Once parsed successfully its possible to easily explore the tree and retrieve any data required. Modifications to the tree are also possible. Memory management is totally transparent through the use of smart pointers so there is no need to call new, delete, malloc or free, decreasing the possibility of memory leaks and increasing its efficiency.

\subsection{Data to Variable Linker}

On request from the rendering engine the data to variable linker will use the RSS configuration file information to request the required RSS data from the XML parser for a given variable name and return the data to the rendering engine.

\subsection{RSS Configuration File}

A new configuration file is created for every RSS feed downloaded from the Internet unless it already exists. The generated configuration file lists all the variables 
found in the RSS feed as a comment, to be used as a reference when editing the configuration file. This does not necessarily include all possible variables as the feed may change over time. For accuracy it is advised to still read about the the particular RSS feed you are attempting to use from its origin. The file needs to be edited to create links between the RSS data and the rendering engine, once this is done it dose not need editing unless the RSS feed changes.

The configuration files are implemented in this way so that its possible to easily change the data being used within the system, it also gives the added advantage of quickly and easily adapting the system to new or changed RSS feeds. An example of the RSS configuration file is given below.

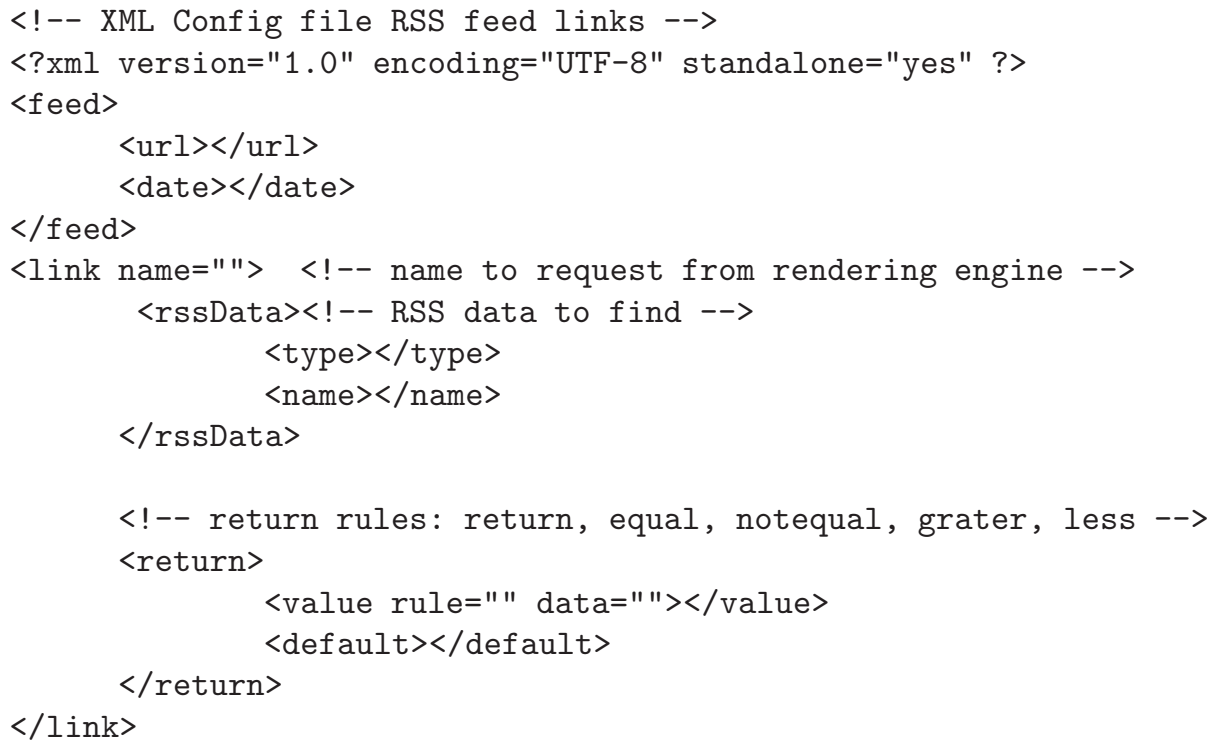

Each link has a name, this would be the name requested from the rendering engine. The rssData values are used to find the RSS data required, type refers to an attribute or node, and name refers to the type name in the RSS feed. It is possible to set up basic rules when returning data, these are return which simply returns the RSS data value, equal, grater, and less, which compare the RSS data to some constant value, and will return the RSS data value or a given return value if the rule is true. If the rule fails or is false a default value is returned instead.

\section{Use Cases}

Accurate weather simulation and prediction can be a complicated and very compute intensive process, by using pre processed weather data that gives the predicted weather, this task of compiling the metrological data into useful information is skipped. There are may sites on the Internet that use RSS feeds for weather prediction information, Yahoo Weather being one of them. This section 
describes the use of a weather RSS feed in conjunction with the described work and a weather rendering engine to produce a virtual environment that's able to display the current weather of a specified geographical location around the world.

\subsection{Rendering Engine}

The rendering engine is able to animate various weather effects including the sun, clouds, rain, and the effect of the wind. The engine has been written in a modular form and is now not limited to these weather types, additional modules can be added to increase the diversity of weather patterns that the engine can simulate. Each module of the rendering engine is able to take parameters associated with their type of weather, the rendering engine currently has nine parameters that are used when rendering all weather simulations and are mentioned in the following sections. All the module parameters are linked with the RSS configuration files mentioned above, and the rendering engine requests the required data from the data to variable linker during runtime.

Rain and Clouds. The rain and cloud objects are able to load a 3D model to be used for each rain drop or cloud rendered in the environment. They are effected by the wind speed and direction, during animation the objects are pushed in the direction of the wind. The objects are randomly scattered over the environment and in the case of clouds are morphed in size to give the appearance of different cloud shapes. The volume of rain and cloud objects rendered related to the parameters passed to the rendering engine and can render from zero to MAX_RAIN or MAX_CLOUD number of objects which is defined at compile time of the application. Transitions between different amounts of rain and clouds are performed gradually over time to give a more realistic weather changing effect.

Sky Background. The entire environment is enclosed in a coloured dome with makes the background sky. The sky colour can be change to any value and will gradually fade to that value from it's current colour over time. During cloudy rainy weather a greyer sky is perceived, where as hot weather will produce a brighter shade of blue.

During sunrise and sunset hours the sky will change accordingly, during the night the sky will darken.

The Sun. The sun uses the current time of day and places itself accurately in the sky according to sunrise and sunset times. The sun will light all of the models in the environment according to its position in the sky. It is also possible to speed up time and see a whole day's cycle.

Fog. Fog was implemented in a novel fashion; a plane is placed in front of the user, and as the user moves the plane moves with them. Different transparencies are set depending on the weather visibility. If the plane is set to be completely transparent, it shows the scene as normal and is not noticed. A semi-translucent plane shows a partially foggy scene and an opaque plane results in a very foggy 
scene. Fog was implemented this way for simplicity, at a slight sacrifice of aesthetic look. Transitions between visibility levels are changed over time to give the effect of fog either emerging or dispersing. Fig 3 shows an example of the simple fog effect.
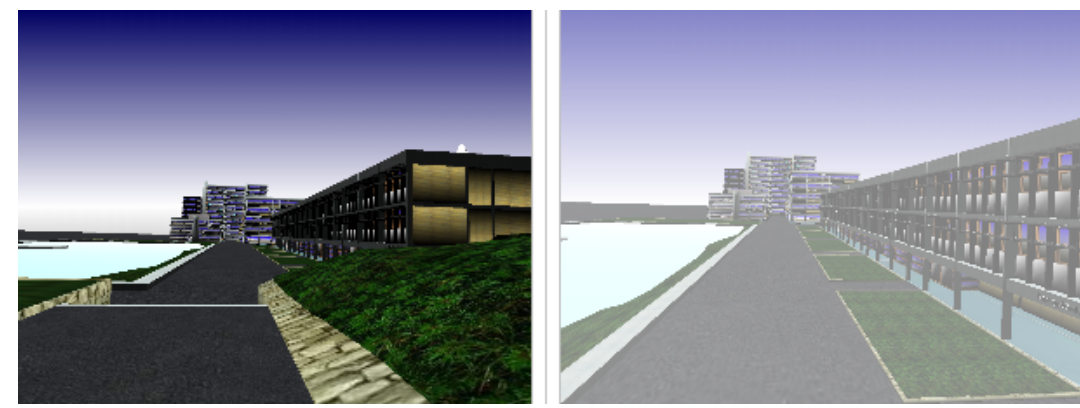

Fig. 3. An example of a scene with and without fog present

Wind. The wind object does not render any graphics but manipulates the rain and cloud objects. The wind speed and direction are converted into a translation that is applied to both the clouds and rain. The wind will randomly deviate slightly from its true speed and direction to more closely resemble wind in the real world as it is never constantly blowing in exactly the same direction or speed all the time.

Time. The time object has two main roles. It either returns the current time of day converted into minutes, or a custom time interval can be set to increase or decrease the speed of time. The time object is used mostly by the sun but can also be used to check for updated RSS feeds after an elapsed time period.

Configuration File. There are many weather feeds available on the Internet today and each is specific to it's source, therefore a system needed to be developed that would allow easy configuration of this received data so it could be easily converted into useful information for the rendering engine.

The applications configuration file allows you to map selected values from a specified RSS feed to the weather parameters needed by the rendering engine. It also stores default values for parameters that cannot be mapped, details of the location the RSS weather data is associated with, proxy details and authentication information if needed, a specific model can be loaded with the weather simulation, as well as custom models for the rain and clouds. The functionality for adding custom files for the rain and cloud models was added for diverse effects like being able to render a scene with it literally raining cats and dogs, or to use a superior cloud model than the one provided. An example of the application configuration file is given below. 


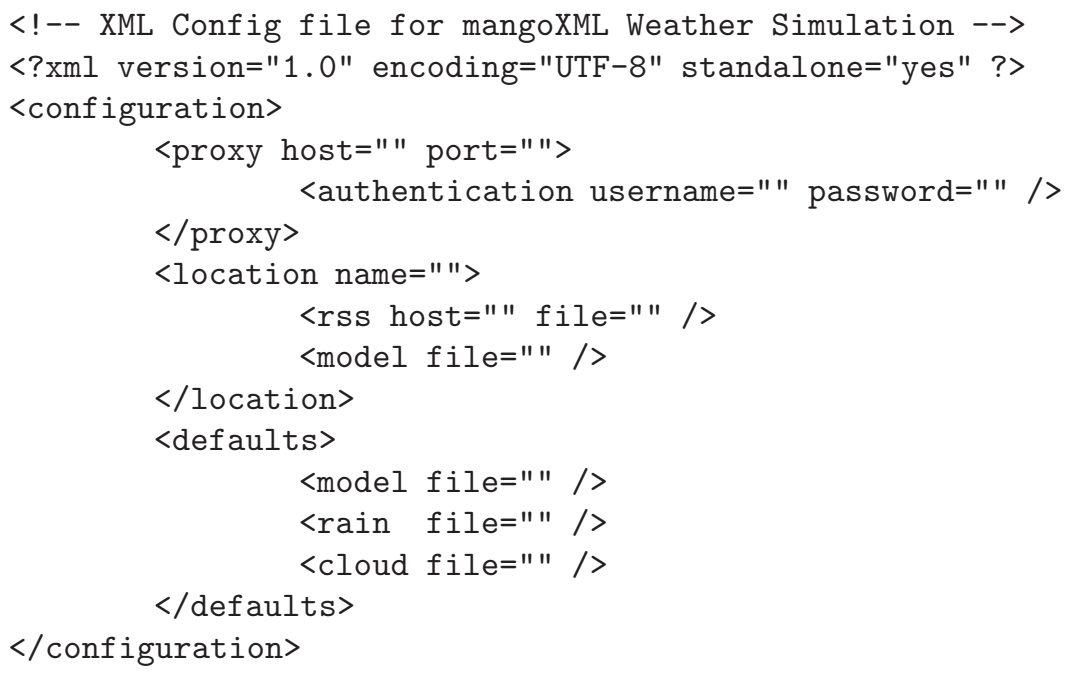

If a proxy isn't used then these details should be removed from the configuration file so that the application knows not to attempt to connect via proxy. If any attributes are left blank, they will be requested during runtime of the application, giving the option of not needing to save usernames and passwords into the configuration file.

\section{Conclusion}

This work demonstrates a novel way of using RSS data from the Internet enabling an easier way to integrate it into an application or virtual environment. The system is completely configurable and very easy to use. With the added ability of being able to connect to the Internet via proxy services using basic authentication methods.

Many applications will benefit from this work as they gain a simple way to incorporate up-to-date RSS feed data into their systems. The work give the ability to dynamically change environment variables from RSS feeds, and enables a vast amount of information to be incorporated into their systems, increasing productivity, and quality.

The case study has been successfully implemented, and the addition of an XML parser and variable linker has increased its usability, giving it the ability to use many different weather sources. The application is also cross platform compatible and able to run on multiple architectures.

\section{Future Work}

Further tests from other RSS sources need to be carried out to show that similar results can be achieved with different RSS feeds. The application still has much more to offer, further development in the XML configuration files could include 
a more generalised and intelligent way to integrate the RSS feed data to the rendering engine so that the configuration files are easier to configure. Incorporating all the defined variables of the rendering engine into the configuration files will allow further changes to the rendering engine without recompiling the source code. Implementing a callback structure for the RSS data linker would allow seemless updates to single variables rather than requesting an update for all variables from the engine. The rendering engine can have many graphic improvements including volumetric clouds and fog. Weather after effects like wet shiny surfaces during and after rainfall. Inclusion of further weather types, snow and hail could easily be produced from a derived rain class. The inclusion of audio would increase the realism of the weather types. The work should also be expanded into other areas of use to enable an easy way of intergrating RSS data into applications, opening them to larger resources of data.

\section{References}

1. Windward Mark Interactive, http://www.windwardmark.net

2. Frank, I., Vanden Berghen: C++ xml parser, http://www.applied-mathematics.net/tools/xmlParser.html

3. Brukakis, Dimitri: Mobile weather for s60 smartphones, http://www.ubahnstation.net/projects/mweather

4. Karelitz, Demiralp, D.B., Cagatay, S.Z., Jackson, C.D., Laidlaw, Cave, D.H.: fishtank virtual-reality displays: A qualitative and quantitative comparison. IEEE Transactions on Visualization and Computer Graphics 12(3), 323-330 (2006)

5. Dunk, A.: Virtual weather. SCARP (2006)

6. Linden Labs. Linden lab shines new light on second life with acquisition of technology from windward mark interactive (May 2007), http://lindenlab.com/press/releases/05_21_07

7. Riley, D.: Better clouds, wind coming to second life (May 2007), http://www. techcrunch.com/2007/05/21/better-clouds-wind-coming-to-second-life/

8. Jeffrey, C., Henrik, M., Nielsen, F., Masinter, L., Leach, P.J., Berners-Lee Roy, T., Fielding, T., Gettys, J.: Hypertext transfer protocol - http/1.1. (June 1999), http://www .w3.org/Protocols/HTTP/1.1/rfc2616.pdf

9. Microsoft Game Studios. Microsoft flight simulator, http://www.fsinsider.com/Pages/default.aspx

10. Lionhead Studios. Black \& white, http://www.lionhead.com/bw/about.html

11. Weber, A.: 3D Weather data visualization in second life (October 2006), http://www . secondlifeinsider.com/2006/10/28/3d-weather-datavisualization-in-second-life/

12. Yahoo! Weather Weather prediction and RSS feeds. http://weather.yahoo.com 